\title{
Efficacy of intravenous amphotericin B-polybutylcyanoacrylate nanoparticles against cryptococcal meningitis in mice
}

This article was published in the following Dove Press journal:

International Journal of Nanomedicine

26 April 201 I

Number of times this article has been viewed

$\operatorname{Nan} X u^{1,2}$

Julin $\mathrm{Gu}^{3}$

Yuanjie Zhu ${ }^{3}$

Hai Wen ${ }^{3}$

Qiushi Ren'

Jianghan Chen ${ }^{3}$

'Institute for Laser Medicine and Biophotonics, School of Life Sciences and Biotechnology, Shanghai Jiao

Tong University, Shanghai, People's Republic of China; ${ }^{2}$ Department of Dermatology, Shanghai East Hospital, Shanghai, People's Republic of China; ${ }^{3}$ Department of Dermatology, Shanghai Changzheng Hospital, Shanghai, People's Republic of China

Correspondence: Qiushi Ren, Institute for Laser Medicine and Biophotonics, School of Life Sciences and Biotechnology, Shanghai Jiao Tong University,

Shanghai 200240, China,

Tel +86021 34204078

Email qsren@sjtu.edu.cn

Jianghan Chen Department of Dermatology, Shanghai Changzheng Hospital, Shanghai 200003, People's

Republic of China

$\mathrm{Tel} / \mathrm{Fax}+8602181885492$

Email chenjinghan@I26.com
Abstract: Amphotericin B deoxycholate $(\mathrm{AmB})$, a classic antifungal drug, remains the initial treatment of choice for deep fungal infections, but it is not appropriate for treatment of cryptococcal meningitis due to its inability to pass through the blood-brain barrier (BBB). We examined the efficacy of amphotericin B-polybutylcyanoacrylate nanoparticles (AmB-PBCANPs) modified with polysorbate 80 that had a mean particle diameter less than 100 nanometers $(69.0 \pm 28.6 \mathrm{~nm})$. AmB-PBCA-NPs were detected in the brain 30 minutes after systemic administration into $\mathrm{BALB} / \mathrm{c}$ mice and had a higher concentration than systemically administered AmB liposome (AmB-L, $P<0.05$ ); AmB was not detected in the brain. Following infection for 24 hours and then 7 days of treatment, the survival rate of mice in the AmB-PBCA-NP group $(80 \%)$ was significantly higher than that of the $\mathrm{AmB}(0 \%)$ or AmB-L $(60 \%)$ treatment groups. Fungal load was also lower when assessed by colony-forming unit counts obtained after plating infected brain tissue $(P<0.05)$. Our study indicates that AmB-PBCA-NPs with polysorbate 80 coating have the capacity to transport $\mathrm{AmB}$ across the $\mathrm{BBB}$ and is an efficient treatment against cryptococcal meningitis in a mouse model.

Keywords: cryptococcal meningitis, polybutylcyanoacrylate (PBCA), nanoparticles, brain targeting

\section{Introduction}

The incidence of deep fungal infection has risen significantly in response to the increased prescription of antibiotics in patients treated with immunosuppressive drugs, or immunosuppression following organ transplantation, malnutrition, or diabetes. ${ }^{1}$ Although amphotericin B deoxycholate (AmB) continues to be the gold standard for treatment by selectively binding to ergosterol in the fungal cell membrane, which results in the leakage of intracellular contents and cellular death, ${ }^{2}$ clinical application of AmB against cryptococcal meningitis is limited due to its inability to pass through the blood-brain barrier (BBB), ${ }^{3,4}$ In addition, serious side effects and toxicity, including acute infusion reactions (nausea, vomiting, rigor, fever, or hypoxia) and dose-dependent nephrotoxicity, limit the use of AmB. ${ }^{2,5}$ Incorporation of drugs into liposomes minimizes toxicity without a loss of pharmacologic effect. ${ }^{6}$ Lipid modifications, including amphotericin B liposome (AmB-L), amphotericin B lipid complex, and amphotericin $\mathrm{B}$ colloidal dispersions, were developed to improve the tolerance profile of $\mathrm{AmB}$ and improve access across the BBB. Compared with the recommendations for $\mathrm{AmB}$, the lipisome-incorporated forms can be administered at a five to seven times higher dosage and contribute to better efficacy, but the frequency of infusion-related adverse events remains a problem. ${ }^{7}$ These issues have necessitated the need for a better drug 
delivery medium that provides permeability to the BBB and minimizes AmB toxicity, as well as effective control of the cryptococcal meningitis infection.

Techniques using drug-nanoparticle complexes have been developed recently as drug delivery systems, and a number of materials have emerged as potential brain-targeting carriers. Polybutylcyanoacrylate (PBCA) has been suggested as a good sustained drug vehicle that potentially allows different substances to pass through the BBB and can be widely used for both treatment and diagnostic purposes via oral or intravenous administration. ${ }^{8-11}$

Compared with some other carriers, PBCA was confirmed to ameliorate BBB permeability by 3 - to 16 -fold. ${ }^{12}$ Kreuter and Gelperina ${ }^{9}$ used PBCA to transport doxorubicin and found that it significantly increased the survival times of rats with cerebral cancer, leading to complete tumor remission in $20 \%-40 \%$ of the animals. The use of nanoparticles also considerably reduced the effective dosage of doxorubicin, which is limited due to its testicular toxicity and cardiotoxicity. ${ }^{9}$ Previous studies have not focused on antifungal agents.

Polysorbate 80 enhances the central nervous system uptake of drug nanoparticles in both human and bovine primary brain capillary endothelial cells, which suggests that it is a preferred surfactant. ${ }^{13,14}$ When coated with polysorbate 80 , the accumulation of nanoparticles in the reticuloendothelial system decreased by $40 \% 1$ hour after injection, ${ }^{11}$ and the drug concentration in the brain increased by 20 -fold. ${ }^{13}$

Previously, we employed polylactic acid-b-polyethylene glycol (PLA-b-PEG) as a way of loading AmB in order to achieve penetration across the $\mathrm{BBB} .{ }^{15}$ In this present study, we prepared AmB-PBCA-nanoparticles (AmB-PBCA-NPs) coated with polysorbate 80 in order to explore another method of encapsulating AmB within the polymer matrix and to determine their effectiveness in permeating through the brain tissue.

\section{Materials and methods Chemicals and reagents}

AmB and AmB-L were ordered from New Pioneer Pharmaceutical Co. Ltd (Shanghai, People's Republic of China). $\alpha$-butyl-cyanoacrylate ( $\alpha$-BCA, Batch No: 20050508) was obtained from Beijing Shunkang Co. Ltd (Beijing, People's Republic of China). Polysorbate 80, Dextran T-70, and acetic acid were purchased from Shanghai Chemical Reagent Co. Ltd (Shanghai).

\section{Preparation of AmB-PBCA-NPs and size determination}

AmB-PBCA-NPs were prepared as follows. Unloaded PBCA-NPs were produced by spontaneous anionic polymerization of $\alpha$-BCA $(20 \mathrm{mg} / \mathrm{mL})$ with $300 \mathrm{mg}$ of Dextran T-70 during magnetic stirring at room temperature for 4 hours. Twenty milligrams of $\mathrm{AmB}$ were dissolved in the mixed solvents for 4 hours, and then $0.1 \mathrm{~mL}$ of polysorbate 80 was added dropwise to $10 \mathrm{~mL}$ of the colloidal solution. After sufficient mixing, the complex was incubated at $37^{\circ} \mathrm{C}$ for at least 2 hours. Prepared AmB-PBCA-NPs were stored at $4^{\circ} \mathrm{C}$ prior to use.

\section{Size distribution and morphology of AmB-PBCA-NP}

The AmB-loaded nanoparticle suspension was homogenized by sonication for 20 minutes. The diameter and size distributions of the particles were determined by a Coulter Laser Granulometer (Zetasizer, Malvern Instruments, Malvern, UK) at room temperature.

After homogenization, a drop of the nanoparticle suspension was loaded onto a copper halftone grid and stained by a $1 \%$ (wt) phosphotungstic acid solution. The sample was characterized by a JEM-100CXII transmission electron microscope (Hitachi, Tokyo, Japan) using an electron beam accelerating voltage of $100 \mathrm{kV}$.

\section{Encapsulation rate of $A m B$ into AmB-PBCA-NPs}

The spectrum of AmB-PBCA-NPs was analyzed and calibrated at a wavelength of $405 \mathrm{~nm}$ (UV-256, Shimadzu, Japan). The encapsulation rate of AmB within the polymer matrix was calculated by the formula: entrapment efficiency $(\%)=($ weight of loaded $\mathrm{AmB}) /($ weight of $\mathrm{AmB}$ input) $\times 100 \%$.

\section{Animals and fungus}

Healthy BALB/c mice weighing $20 \pm 5 \mathrm{~g}$ (provided by the Laboratory Animal Center of Academia Sinica, Shanghai, People's Republic of China) were used in the biodistribution and therapeutic studies. All mice were housed in a pathogenfree environment and received sterilized food and water. All procedures complied with the Guide for the Care and Use of Laboratory Animals.

The Cryptococcus neoformans 30629B suspension used for the inoculation was prepared by diluting the fungus with sterile saline to a final concentration of $5.0 \times 10^{8}$ cell $/ \mathrm{mL}$, 
which was determined using a hemocytometer and microscopic observation.

\section{Chromatography}

Analytical high-performance liquid chromatography (HPLC) with an ultrabase $\mathrm{C}_{18}$ reversed-phase column (Dikma, Japan) was used for chromatographic analysis (mobile phase: acetonitrile-water [40:60] acetic acid [4\%], flow rate: $0.9 \mathrm{~mL} /$ minute, ultraviolet detection wavelength: $405 \mathrm{~nm}$, and column temperature: $37^{\circ} \mathrm{C}$ ).

\section{Pharmacokinetic studies}

An injection of AmB (1 mg/kg), AmB-PBCA-NPs (3 mg/kg), or AmB-L $(10 \mathrm{mg} / \mathrm{kg})$ was administered to mice through the tail vein. Data were collected at 0.5 hours, 1 hour, 3 hours, 6 hours, 12 hours, 24 hours, and 48 hours ( $n=28$ /group, $n=4 /$ time point). The thorax was opened and the animals perfused through the heart with saline to wash out the remaining AmB. Tissues were then homogenized with sterile saline, and the supernatant of the homogenate was extracted with an equal volume of acetonitrile (for the brain: $1 \mu \mathrm{g} / \mathrm{mL}$; for the heart, lung, liver, and kidney: $2 \mu \mathrm{g} / \mathrm{mL}$ ). The organic phase was analyzed by HPLC with quercetin as an internal standard.

\section{Effect of AmB-PBCA-NPs against murine cryptococcal meningitis}

$\mathrm{BALB} / \mathrm{c}$ mice were anesthetized with ethyl ether and then inoculated with the $C$. neoformans suspension (concentration $5 \times 10^{8}$ cell $/ \mathrm{mL}$, dose $5 \mu \mathrm{L} / 20 \mathrm{~g}$ ) through foramen magnum 24 hours after an intraperitoneal injection of cyclophosphamide ( $2 \mu \mathrm{g} / 20 \mathrm{~g}$; Jiangsu Hengrui Medical Co. Ltd, Jiangsu, People's Republic of China; H 32020857).

Twenty-four hours after the fungal inoculation, an injection of i) AmB (1 mg/kg), ii) AmB-PBCA-NPs (3 mg/kg), iii) AmB-L (10 mg/kg), or iv) saline was administered through the tail vein and then daily injections maintained for 7 consecutive days. Twenty-four hours after the first injection (day 1) and last injection (day 7), the mice $(n=10 /$ group, $n=5 /$ time point) were sacrificed and the brains were dissected, weighed, and homogenized with sterile saline $(2 \mathrm{~mL} / \mathrm{g})$. The homogenate was serially diluted with sterile saline, then $0.1 \mathrm{~mL}$ of the suspension was inoculated onto sabouraud dextrose agar plates and incubated at $37^{\circ} \mathrm{C}$ for 24 hours and the number of colonies counted.

In a separate experiment, the mice were inoculated with the $C$. neoformans suspension and 24 hours later given daily injections of i) AmB (1 mg/kg), ii) AmB-PBCA-NPs (3 mg/kg), iii) AmB-L (10 mg/kg), or iv) saline for 7 days. The survival rates of the mice ( $n=20$ /group) were observed for 20 days after the beginning of treatment.

\section{Routine blood tests to determine the effect of treatment on the liver, kidney, and blood components}

Mice were injected daily via the tail vein with i) $A m B$ (1 mg/kg), ii) AmB-PBCA-NPs (3 mg/kg), iii) PBCA-NPs (blank nanoparticles without $\mathrm{AmB}, 3 \mathrm{mg} / \mathrm{kg}$ ), and iv) polysorbate $80(1 \%)(n=10 /$ group$)$. Polysorbate 80 replaced saline as a control in order to verify that it had no effect on the general health of the animals. Orbital blood was collected 1 day before, 1 day after, or 7 days after drug administration. Blood indices were determined for erythrocytes (red blood cells [RBCs]), leucocytes (white blood cells [WBCs]), and platelets (PLTs) using an automatic hemocytometer. Whole blood was centrifuged at $14000 \mathrm{rpm}$ for 10 minutes and stored at $-20^{\circ} \mathrm{C}$ prior to routine tests for liver function using alanine aminotransterase (ALT) and aspartate aminotransferase (AST), and kidney function using blood urea nitrogen (BUN) and creatinine $(\mathrm{Cr})$.

\section{Statistical analysis}

All the aforementioned experiments were repeated three times. The differences between experimental groups were analyzed by analysis of variance with a post hoc test. All values are presented as the mean \pm standard deviation, and results were considered statistically significant with $P \leq 0.05$.

\section{Results \\ Characteristics of AmB-PBCA-NPs}

The mean diameter of the prepared AmB-PBCA-NPs was $69.0 \pm 28.6 \mathrm{~nm}$, and they were spherical in shape (Figure 1). After polysorbate 80 modification, the nanoparticles were slightly less spherical, but the sizes of the particles were not significantly different. The mean encapsulation rate of AmB-PBCA-NPs was $56.0 \% \pm 1.4 \%$.

\section{Pharmacokinetic study}

The concentration of AmB in tissues was determined by HPLC with quercetin as an internal standard (Figure 2). The detection limit of $\mathrm{AmB}$ was $10 \mathrm{ng} / \mathrm{mL}$, and the interday and intraday coefficients of variation were $5 \%$ or less between $5 \mathrm{ng} / \mathrm{mL}$ and $200 \mathrm{ng} / \mathrm{mL}$. 

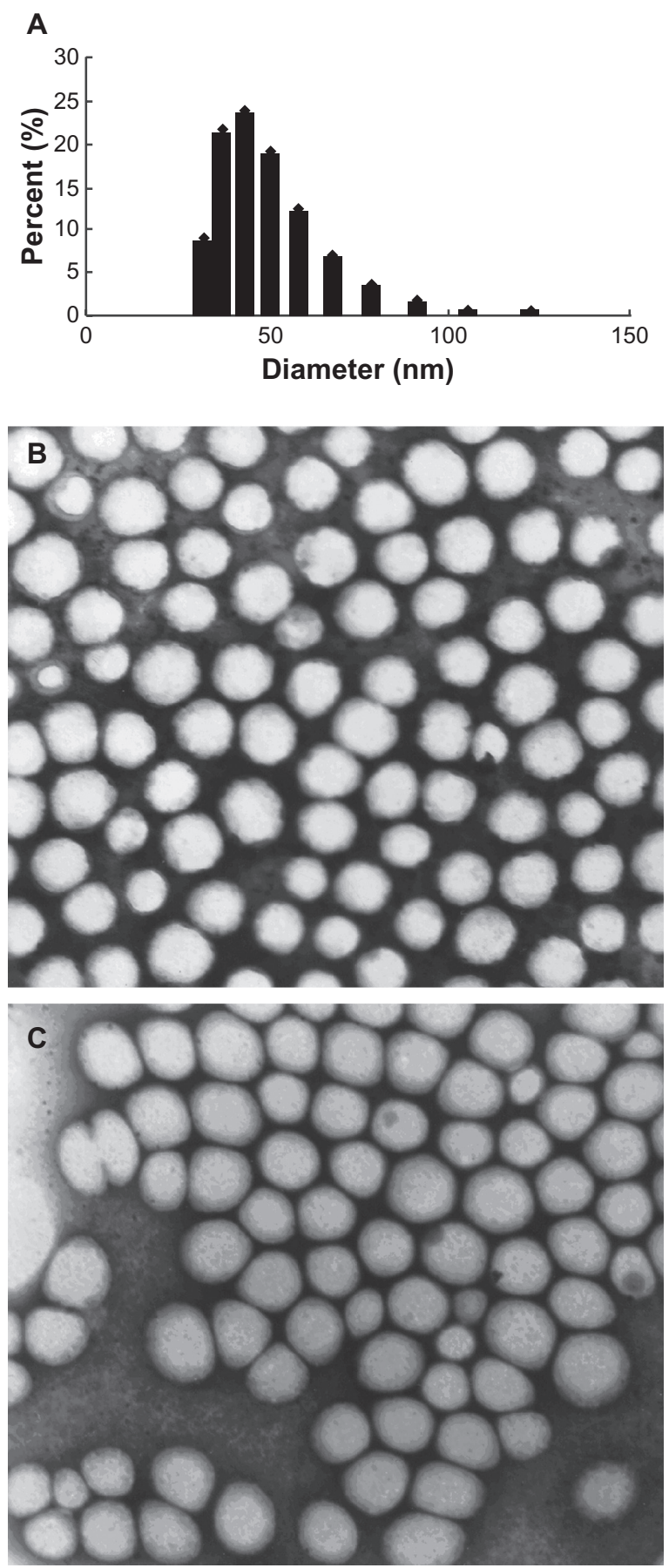

Figure I Size distribution and morphology of amphotericin B-polybutylcyanoacrylate nanoparticles (AmB-PBCA-NPs). A) The mean diameter of polysorbate 80-coated AmB-PBCA-NPs determined by a Coulter Laser Granulometer was $69.0 \pm 28.6 \mathrm{~nm}$. B) AmB-PBCA-NPs without polysorbate 80 coating were spherical in shape. C) AmB-PBCA-NPs after polysorbate 80 modification had a more elliptical shape but were of a similar size to uncoated particles $(\times 50,000)$.

$\mathrm{AmB}$ was not detected in the brains of AmB-treated mice. The drug concentration in the brain tissue of the AmB-PBCA-NP group was present within 0.5 hours after injection and reached a peak value of $133 \pm 4 \mathrm{ng} / \mathrm{g}$ by 3 hours and then gradually fell to $66.1 \pm 1.1 \mathrm{ng} / \mathrm{g}$ by 48 hours after

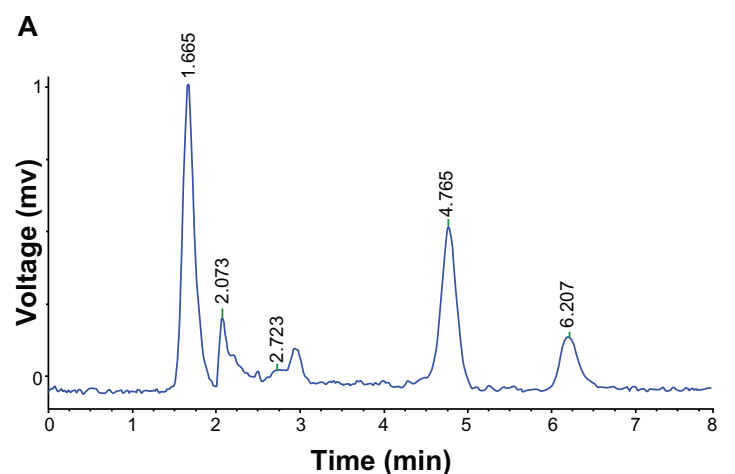

B

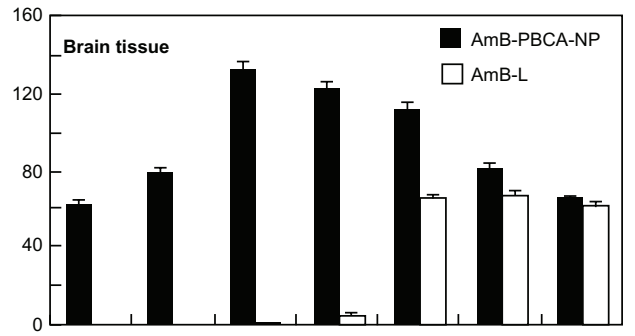

C

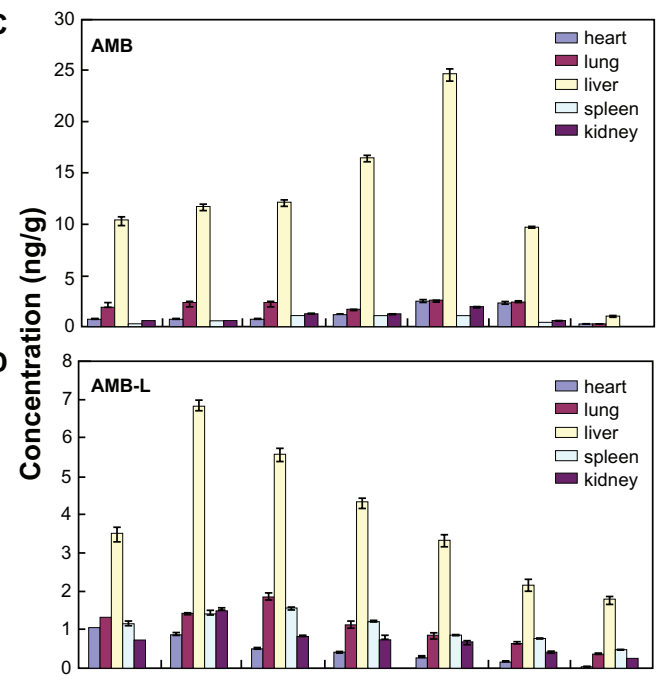

E

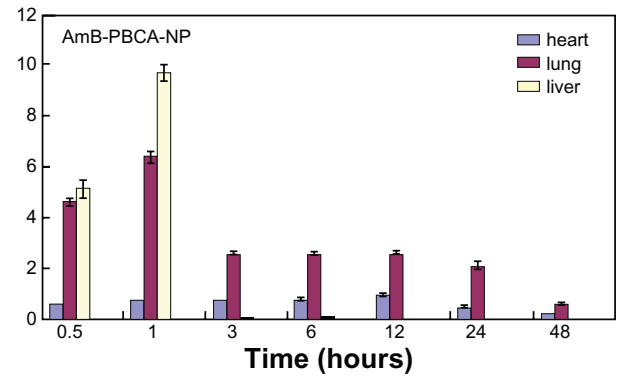

Figure 2 Amphotericin $B(A m B)$ levels in various tissues following injections of AmB, amphotericin B-polybutylcyanoacrylate nanoparticles (AmB-PBCA-NPs), or amphotericin $B$ liposome (AmB-L). A) High-performance liquid chromatogram showing the presence of $A m B$ in brain tissue. The detection limit of $A m B$ was $10 \mathrm{ng} / \mathrm{mL}$, and the interday and intraday coefficients of variation were $5 \%$ or less between $5 \mathrm{ng} / \mathrm{mL}$ and $200 \mathrm{ng} / \mathrm{mL}$. B) AmB was not detected in the brain tissue of mice treated with noncomplexed $A m B$ but was found 30 minutes after AmB-PBCA-NP treatment and 3-6 hours after AmB-L treatment ( $n=28 /$ group). C-D) The concentration of $A m B$ in various tissues is shown after AmB, AmB-L, and AmB-PBCA-NP treatment, respectively $(n=28)$. AmB was concentrated mainly in the liver; note also the higher lung concentration when using AmBPBCA-NPs. 
the injection. In contrast, $\mathrm{AmB}$ was not detected until 3 hours after the injection in the brains of the AmB-L-treated mice and reached a peak of $67.2 \pm 1.9 \mathrm{ng} / \mathrm{g}$ at 24 hours before falling to slightly lower values at 48 hours $(61.1 \pm 1.6 \mathrm{ng} / \mathrm{g})$. Peak values of AmB in AmB-PBCA-NP-treated mice were significantly higher than peak values in AmB-L-treated mice ( $P<0.01, \mathrm{n}=28$ /group) (Figure 3 ), although AmB concentration was not significantly different by 48 hours.

The concentration of AmB was highest in liver tissue and gradually increased, reaching a peak at 12 hours (24.6 $\pm 0.5 \mu \mathrm{g} / \mathrm{g}, \mathrm{n}=28$ /group) before falling to lower levels at 48 hours. Lower levels of AmB were seen in the heart, lung, spleen, and kidney $(<3 \mu \mathrm{g} / \mathrm{g})$, although the concentration of $\mathrm{AmB}$ was noticeably higher in lung and heart tissue between 12 hours and 24 hours. In contrast, with the exception of the heart, the concentration of AmB-L showed a much earlier increase in all tissue and peaked between 1 hour and 3 hours before falling to lower levels at 48 hours. Similar to AmB, the concentration of AmB-L was highest in the liver and peaked at 1 hour $(6.8 \pm 0.1 \mu \mathrm{g} / \mathrm{g})$ after the injection and considerably earlier when compared with that seen in the brain tissue (1 hour vs 12 hours), albeit at a much lower concentration. AmB-L concentration in the heart steadily declined from that seen at 0.5 hours. The concentration of AmB-PBCA-NPs also showed an early peak in the liver tissue 1 hour after the injection $(9.7 \pm 0.3 \mu \mathrm{g} / \mathrm{g})$ but fell more rapidly to very low levels at 3 hours compared with AmB-L. The peak also occurred earlier than that observed in the brain tissue but, unlike that seen in the brain tissue, was not sustained over the next 48 hours. In contrast to AmB and AmB-L, AmBPBCA-NP levels were noticeably higher in lung tissue, with a peak $(6.3 \pm 0.4 \mu \mathrm{g} / \mathrm{g})$ at 1 hour after the injection, but were absent in spleen and kidney tissue (Figure 4).

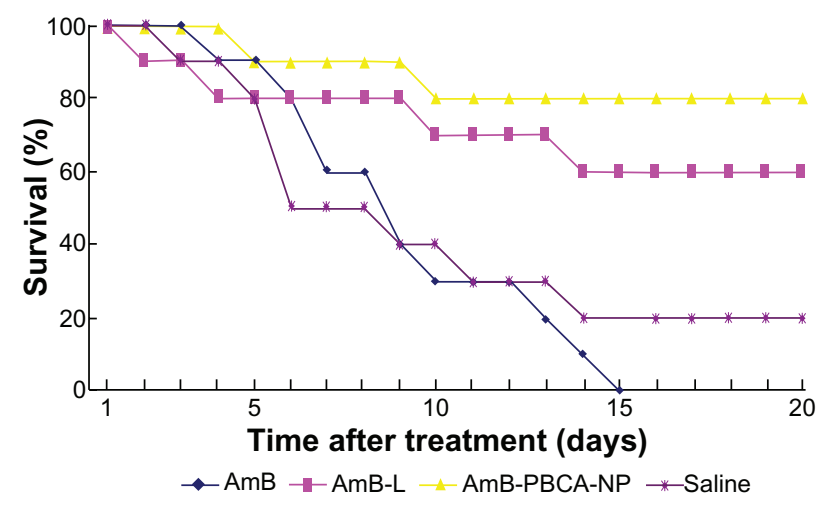

Figure 3 Survival rate of mice under different treatments. All amphotericin B $(\mathrm{AmB})$-treated mice died within 15 days. In contrast, $80 \%$ of the mice survived after amphotericin B-polybutylcyanoacrylate nanoparticle (AmB-PBCA-NP) injections, and $60 \%$ survived after amphotericin $B$ liposome (AmB-L) treatment $(n=20 /$ group $)$.

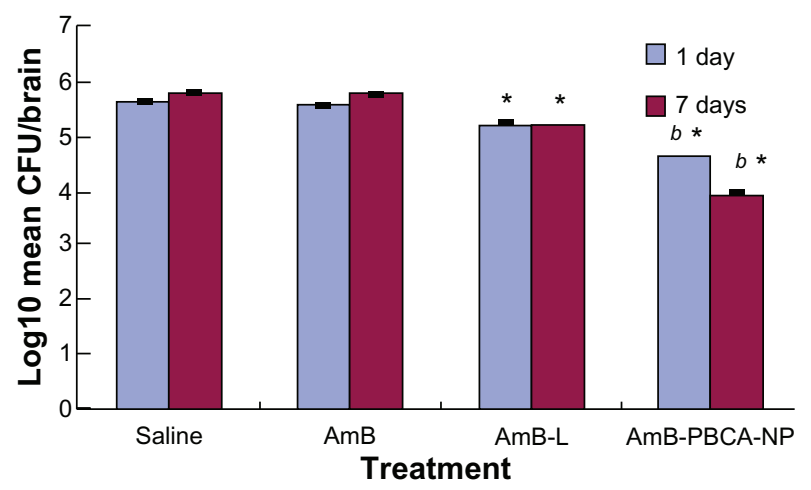

Figure 4 Fungal load in the brain of Balb/c mice infected with Cryptococcus neoformans treated with amphotericin $B(A m B)$, amphotericin B liposome (AmB-L), amphotericin B-polybutylcyanoacrylate nanoparticles (AmB-PBCA-NPs), or saline. Notes: Data were assessed from plated brain tissue and the number of colonyforming units (CFU). $* P<0.05$, value vs $A m B$ group; ${ }^{b * P}<0.05$, value vs $A m B-L$ group ( $n=10 /$ group)

\section{Therapeutic efficacy of AmB-PBCA-NPs against murine cryptococcal meningitis}

Mice inoculated with $C$. neoformans began to die 3 days after the injection, and the majority of animals had died by day 6 , reaching a maximum on day $14(80 \%)$ without further death until day 20 (Figure 3). Inoculated animals treated with AmB showed a similar trend, although were slightly delayed compared with the saline-treated mice, and the majority of animals were dead by day 10 . Unlike the saline-treated mice, all of those treated with $A m B$ had succumbed to the infection by day 15 . In contrast, the AmB-L treatment group showed a slower decline in numbers beginning on day 2 and lasting until day 14 , with a maintained $60 \%$ survival rate thereafter. Death did not occur until day 4 in mice treated with AmB-PBCA-NPs, and there was a slower decline in numbers until day 10 , with a maintained survival rate of $80 \%$ for the remainder of the experiment.

\section{C. neoformans burden in the brain}

The amount of $C$. neoformans remaining in the brain after 1 day and 7 days of treatment was assessed and expressed as $\log _{10}$ (mean colony-forming units/brain in each group \pm standard error). AmB-treated animals showed a significantly lower $(P<0.05)$ fungal load on day 1 compared with saline-injected animals, but this had increased by 7 days after the consecutive injection and was not different from that seen in the saline-injected animals (Figure 4). AmB-Ltreated animals had significantly lower $(P<0.05)$ fungal loads for day 1 and day 7 compared with the saline- and AmB-treated animals, but they were significantly higher $(P<0.05)$ than those seen in AmB-PBCA-NP-treated animals. AmB-PBCA-NP treatment was clearly the most 
effective in reducing fungal loads on both days compared with saline injections ( 1 day: $4.61 \pm 0.01$ vs $5.63 \pm 0.07$; 7 days: $3.93 \pm 0.07$ vs $5.74 \pm 0.05$, respectively).

\section{Blood component analysis}

In order to investigate the adverse effects of different treatments, various indices were measured for the liver, kidney, and blood system (see Figures 5 and 6). After AmB administration, WBC and PLT counts showed a significant increase by day 7 compared with preinjection
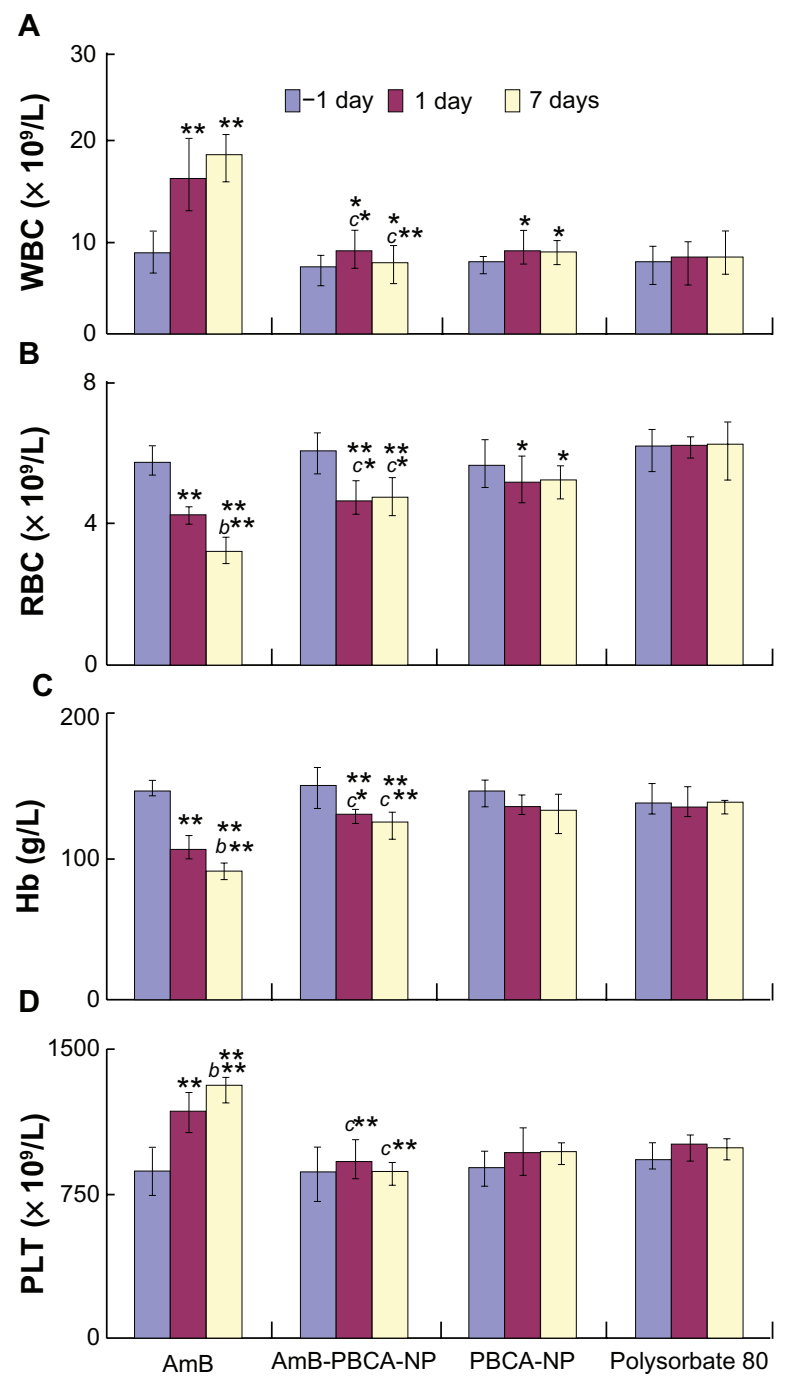

Figure 5 Whole-blood analysis after treatment. Mice were injected daily via the tail vein with i) amphotericin $B(A m B)(I \mathrm{mg} / \mathrm{kg})$, ii) polysorbate 80 -coated amphotericin B-polybutylcyanoacrylate nanoparticles (AmB-PBCA-NPs) (3 $\mathrm{mg} / \mathrm{kg}$ ), iii) blankPBCA-NPs (3 mg/kg), and iv) polysorbate 80 (1\%). Indices: A) White blood cell count $(\mathrm{WBC})\left(\times 10^{9} / \mathrm{L}\right)$; B) Red blood cell count $(\mathrm{RBC})\left(\times 10^{12} / \mathrm{L}\right)$; C) hemoglobin $(\mathrm{Hb})(\mathrm{g} / \mathrm{L})$; D) platelet count $(\mathrm{PLT})\left(\times 10^{9} / \mathrm{L}\right)$.

Notes: ${ }^{* * P}<0.01$; $* P<0.05$, value vs $-\mathrm{I}$ day; ${ }^{b * *} P<0.0$ I, value vs that at I day; ${ }^{c * *} P<0.01$, AmB value vs Amb-PCNB-NP value on the same day; ${ }^{*} P P<0.05$, $A m B$ value vs Amb-PCNB-NP value on the same day. Values are given as the mean \pm standard deviation $(n=10 /$ group $)$.
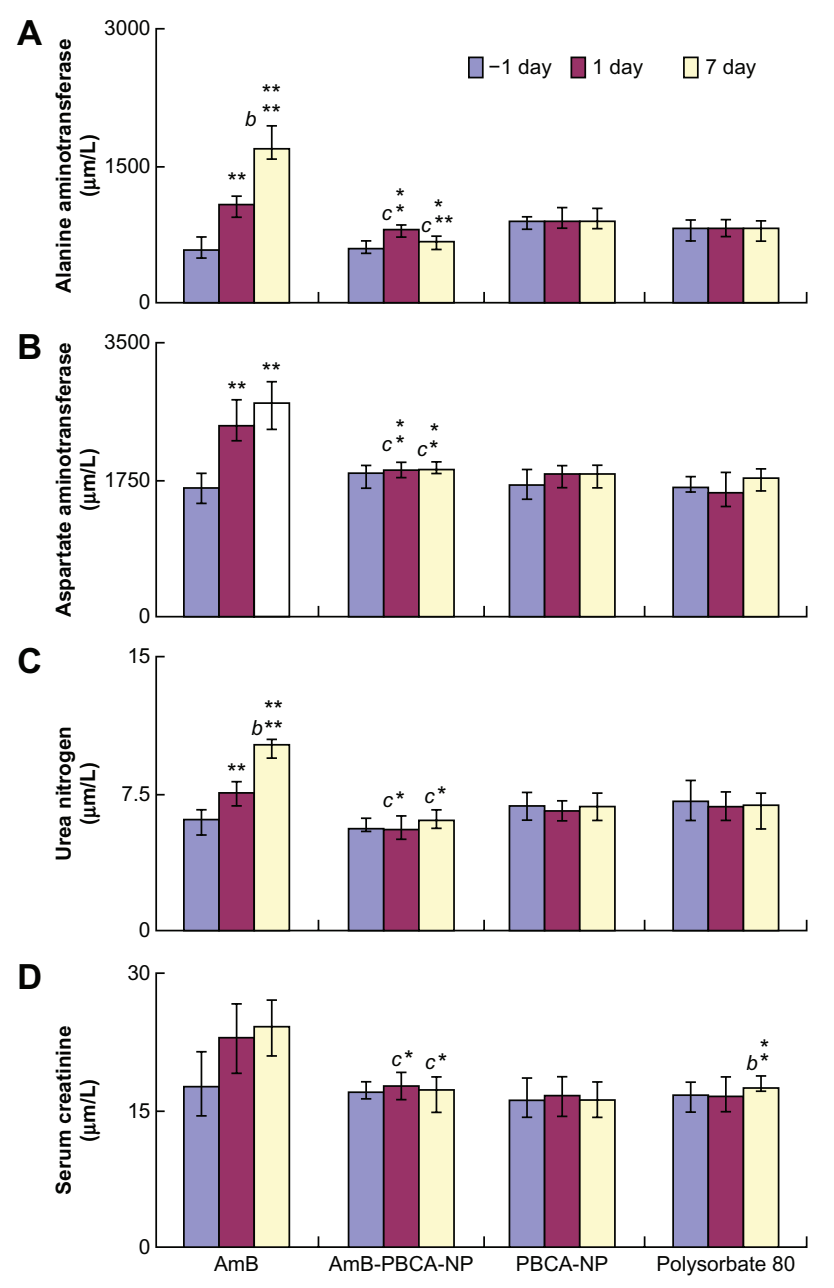

Figure 6 Tests for liver and kidney function. A) Serum levels of alanine aminotransferase and $\mathbf{B}$ ) aspartate aminotransferase as an indicator of liver function. C) Serum levels of blood urea nitrogen and $\mathbf{D}$ ) serum creatinine as an indicator of kidney function.

Notes: ${ }^{*} * P<0.0$ I; $* P<0.05$, value vs - I day; ${ }^{b * * P}<0.0$ I, value vs that at I day; $c * * P<0.01$, amphotericin $B(A m B)$ value vs amphotericin B-polybutylcyanoacrylate nanoparticle (Amb-PCNB-NP) value on the same day; ${ }^{c * P}<0.05, A m B$ value vs Amb-PCNB-NP value on the same day. Values are given as the mean \pm standard deviation ( $n=10 /$ group).

values $(-1$ day; $P<0.01)$. WBC counts were also elevated on days 1 and 7 when animals were treated with Amb-PBCA-NPs and PBCA-NPs $(P>0.05)$ but to a lesser degree compared with $\mathrm{AmB}$; polysorbate 80 had no effect on WBC counts. In contrast, AmB-PBCA-NPs, PBCA-NPs, and polysorbate 80 treatments had no effect on PLT counts. RBC counts were significantly lower on days 1 and 7 compared with pretreatment $(P<0.01)$ for AmB, AmB-PBCA-NPs, and PBCA-NPs, with AmB having the largest effect. Similarly, hemoglobin $(\mathrm{Hb})$ values were significantly lower in the AmB and AmB-PBCA-NP treatment groups $(P<0.01)$, but $\mathrm{Hb}$ was not affected by PBCA-NP or polysorbate 80 treatments. The significant 
differences between AmB and AmB-PBCA-NP treatment groups $(P<0.05)$ indicate that encapsulation by PBCANPs effectively reduced the side effects of $\mathrm{AmB}$ on the blood system.

ALT and AST values, indicative of liver function, were significantly higher on days 1 and 7 following injections of AmB $(P<0.01)$ and AmB-PBCA-NPs $(P<0.05)$ compared with the preinjection values. AmB values were also significantly higher compared with AmB-PBCA-NPs $(P<0.05)$. In contrast, PBCA-NPs and polysorbate had no effect on either ALT or AST values, which suggests that the noted changes are due to AmB itself.

A similar situation was found when measuring the kidney indicators BUN and $\mathrm{Cr}$, in that BUN was significantly higher after $\mathrm{AmB}$ treatment compared with the preinjection values $(P<0.01)$, although no change was seen after AmBPBCA-NP, PBCA-NP, or polysobate injections. Cr values were also higher following $\mathrm{AmB}$ treatment but, due to the variability in the samples, were not significant. Although $\mathrm{Cr}$ values were significantly $(P<0.05)$ higher on day 7 following polysorbate 80 injections, these values still appear to be within the variability of preinjection values in other animals and therefore may not represent a real change in kidney function in these animals.

\section{Discussion}

The clinical application of $\mathrm{AmB}$ is limited by its cytotoxicity. More importantly, impermeability across the BBB results in a low therapeutic efficacy for the treatment of cryptococcal meningitis. ${ }^{16}$ To overcome these problems, intrathecal injection was developed clinically, but most patients in China are reluctant to pursue this treatment due to the risks involved and the inconvenience. Several substitutes for AmB have been commercialized in a search for better therapeutic effects and a safer application. ${ }^{17,18}$

PBCA nanoparticles have been intensively investigated since the first study in 1995, showing that nanoparticles coated with the nonionic surfactant polysorbate 80 are able to cross the BBB. ${ }^{19}$ Recent reports have confirmed that PBCA is an excellent brain-targeting medium with which to deliver therapeutic or diagnostic agents. ${ }^{12}$ We successfully prepared AmB-PBCA-NPs coated with polysorbate 80. Electron microscopy showed the drug-nanoparticle complex had a uniform diameter that was less than $100 \mathrm{~nm}$ (Figure 1), which meets the pharmaceutical requirements for therapeutic usage. Delayed-release tendency tests in vitro showed the drug could be released slowly within 24 hours (data not shown).
$\mathrm{AmB}$ was not detected in the brain, indicating that $\mathrm{AmB}$ on its own does not readily cross the BBB. After encapsulation by PBCA and polysorbate 80 modification, the drug concentration in the brain was detectable 30 minutes after administration and increased to maximum by 3 hours (133 $\pm 4 \mathrm{ng} / \mathrm{g}$ ), which was significantly higher than that of AmB-L. In the previous study, we successfully prepared AmB-PLA-b-PEG-NPs with a polysorbate 80 modification. HPLC data showed that it had a lower concentration in brain tissue than AmB-PBCA-NPs 3 hours after the injection $(126.1 \pm 3.5$ vs $132.5 \pm 3.7, P=0.883>0.05)$, with the peak concentration appearing at 12 hours $(160.4 \pm 3.5$ vs $111.6 \pm 3.3, P=0.018<0.05)$. Although the mechanism by which these complex nanoparticles cross the BBB has not been fully elucidated, many studies have given a number of possible mechanisms. Ramge et $\mathrm{al}^{13}$ suggested that it could be attributed to endocytosis by endothelial cells of the blood vessels within the brain parenchyma. Kreuter ${ }^{20}$ found that apolipoprotein $\mathrm{E}$ (ApoE) plays a vital role in the transport of low-density lipoproteins into the brain, and polysorbate 80 acts mainly as an anchor for the Apo-coated nanoparticles. Therefore, the nanoparticles mimic low-density lipoproteins and interact with the brain capillary endothelial cells, finally transferring the drug into the brain via receptor-mediated endocytosis. A recent study examined the effectiveness of other apolipoproteins, such as ApoE3, B-100, and A-I, in brain targeting, ${ }^{21}$ showing the existence of more than one nanoparticle-brain endothelium interaction mechanism. We also hypothesize that, due to the small size of the complex particles, many of them escape uptake by phagocytic cells of the liver, spleen, and reticuloendothelial system and may account for the significantly lower accumulation of AmB found in these tissues compared with noncomplexed $\mathrm{AmB}$ (Figure 2). The absence of AmB-PBCA-NPs in kidney tissue may be explained as a result of a lower filtration rate through the renal tubules.

Most mice with cryptococcal meningitis started to die 2-4 days after the spore challenge with a $20 \%$ survival rate after 20 days. Animals treated with AmB-PBCA-NPs exhibited improved clinical status (such as appetite, fur color, and condition; data not shown), a higher survival rate, and a significant reduction in the burden of $C$. neoformans remaining in the brain 1 week after the cessation of therapy. A similar survival rate $(80 \%)$ and fungal burden were observed in AmB-b-PEG-NP-treated mice. ${ }^{15}$ AmB-L treatment was also efficacious, but to a lesser degree compared with AmBPBCA-NP treatment. The high death rate of infected mice 
with AmB treatment may be due to the dosage of $1 \mathrm{mg} / \mathrm{kg}$, which is likely to cause the side effects previously mentioned. This is also reflected in the blood analysis data where AmB had a significant effect on all the tested parameters. Indeed, it was noted that injections of AmB must be given very slowly or the death of the animal results, which did not happen with AmB-PBCA-NP administration. Good encapsulation by the nanovector allows sustained drug release over a period of hours, preventing a rapid rise in plasma concentration.

PBCA-NPs were first prepared by Couvreur et $\mathrm{al}^{22}$ in 1979 and were considered to have relatively low toxicity. Moreover, PBCA is among the most rapidly biodegrading synthetic polymers that undergo in vivo degradation by enzymatic ester hydrolysis, producing a primary alcohol-, butanol-, and water-soluble poly-2-cyanoacrylic acid. As a result of this rapid degradation, as well as the low molecular weight of the polymer in the nanoparticle, the polymer material is rapidly eliminated from the body. The main advantages of using PBCA-NPs for brain targeting include their ability to deliver AmB to the brain without changing the drug's original characteristics, as well as to decrease drug escape in the brain and reduce peripheral toxicity. AmB was encapsulated into nanoparticles evenly and stably and released gradually. Consequently, the direct interaction of AmB and cholesterol (similar constitution to ergosterol on the surface of erythrocytes) attenuated accordingly. Thus, the toxicity to erythrocytes was significantly mitigated. In our study, although the side effects were evidently weakened, there were still some changes in the blood system indices and liver function after AmB-PBCA-NP injection, which mainly arise because of the release of the encapsulated AmB.

\section{Conclusion}

In conclusion, our study shows that PBCA can be used as an alternative delivery vehicle for the antifungal agent AmB. In addition, polysorbate 80 is necessary for efficient brain targeting. More importantly, AmB-PBCA-NPs are able to reduce the toxicity of $\mathrm{AmB}$ to the liver, kidney, and blood system and increase the survival rates of mice with cryptococcal meningitis. The next challenge is to further design these drugs to ensure a balance between drug release and drug potency without tissue toxicity and to expand their applicability to fighting other forms of infection.

\section{Acknowledgments}

This research was supported by the National Basic Research Program of China (No. 2011CB707504, No. 2010CB933903), the Shanghai Commission for Science and Technology, Shanghai, People's Republic of China (No. 09 JC1411902), the "111" project from Ministry of Education, People's Republic of China (No. B08020), and the Ministry of Health of the General Logistics Department (10Ma022,2010-04). The authors thank Dr T FitzGibbon for comments and suggestions on earlier drafts of the manuscript.

\section{Disclosure}

The authors report no conflicts of interest in this work.

\section{References}

1. Hunter PR, Nichols G. Epidemiology and clinical features of cryptosporidium infection in immunocompromised patients. Clin Microbiol Rev. 2002;15(1):145-154.

2. Laniado-Laborín R, Cabrales-Vargas MN. Amphotericin B: side effects and toxicity. Rev Iberoam Micol. 2009;26(4):223-227.

3. Pathak A, Pien FD, Carvalho L. Amphotericin B use in a community hospital, with special emphasis on side effects. Clinic Infect Dis. 1998; 26(2):334-338.

4. Ostrosky-Zeichner L, Mart KA, Rex JH, Cohen SH. Amphotericin B: time for a new "gold standard". Clin Infect Dis. 2003;37(3):415-442.

5. Tonomura Y, Yamamoto E, Kondo C. Amphotericin B-induced nephrotoxicity: characterization of blood and urinary biochemistry and renal morphology in mice. Hum Exp Toxicol. 2009;28(5):293-300.

6. Ramaswamy M, Peteherych KD, Kennedy AL, Wasan KM. Amphotericin B lipid complex or amphotericin B multiple-dose administration to rabbits with elevated plasma cholesterol levels: pharmacokinetics in plasma and blood, plasma lipoprotein levels, distribution in tissues, and renal toxicities. Antimicrob Agents Chemother. 2001;45(4):1184-1191.

7. Subirà M, Martino R, Gómez L, et al. Low-dose amphotericin B lipid complex vs conventional amphotericin B for empirical antifungal therapy of neutropenic fever in patients with hematologic malignancies: a randomized, controlled trial. Eur J Haematol. 2004;72(5): 342-347.

8. Kulkarni PV, Roney CA, Antich PP. Quinoline-n-butylcyanoacrylatebased nanoparticles for brain targeting for the diagnosis of Alzheimer's disease. Wiley Interdisciplinary Reviews: Nanomedicine and Nanobiotechnology. 2010;2(1):35-47.

9. Kreuter J. Gelperina S. Use of nanoparticles for cerebral cancer. Tumori. 2008;94(2):271-277.

10. Das D, Lin S. Double-coated poly (butylcynanoacrylate) nanoparticulate delivery systems for brain targeting of dalargin via oral administration. J Pharm Sci. 2005;94(6):1343-1353.

11. Ambruosi A, Yamamoto H, Kreuter J. Body distribution of polysorbate- 80 and doxorubicin-loaded [14C] poly(butyl cyanoacrylate) nanoparticles after i.v. administration in rats. J Drug Target. 2005;13(10):535-542.

12. Kuo YC, Su FL. Transport of stavudine, delavirdine, and saquinavir across the blood-brain barrier by polybutylcyanoacrylate, methylmethacrylate-sulfopropylmethacrylate, and solid lipid nanoparticles. Int J Pharmaceut. 2007;340(1-2):143-152.

13. Ramge P, Unger RE, Oltrogge JB. Polysorbate- 80 coating enhances uptake of polybutylcyanoacrylate (PBCA)-nanoparticles by human and bovine primary brain capillary endothelial cells. Eur J Neurosci. 2000;12(6):1931-1940.

14. Kreuter J, Ramge P, Petrov V. Direct evidence that polysorbate-80-coated poly(butylcyanoacrylate) nanoparticles deliver drugs to the CNS via specific mechanisms requiring prior binding of drug to the nanoparticles. Pharmaceut Res. 2003;20(3):409-416.

15. Ren TB, Xu N, Cao C. Preparation and therapeutic efficacy of polysorbate-80-coated amphotericin B/PLA-b-PEG nanoparticles. J Biomater Sci Polym Ed. 2009;20(10):1369-1380. 
16. Gallis HA, Drew RH, Pickard WW. Amphotericin B:30 years of clinical experience. Rev Infect Dis. 1990;12:308-329.

17. Baginski M, Czub J. Amphotericin B and its new derivatives: mode of action. Curr Drug Metab. 2009;10(5):459-469.

18. Barratt G, Bretagne S. Optimizing efficacy of amphotericin B through nanomodification. Int J Nanomedicine. 2007;2(3):301-313.

19. Kreuter J, Alyautdin RN, Kharkevich DA. Passage of peptides through the blood-brain barrier with colloidal polymer particles (nanoparticles). Brain Res. 1995;674(1):171-174.
20. Kreuter J. Application of nanoparticles for the delivery of drugs to the brain. Int Congr Ser. 2005;1277:85-94.

21. Kreuter J, Hekmatara T, Dreisa S, et al. Covalent attachment of ApoA-I and ApoB-100 to albumin nanoparticles enables drug transport into the brain. J Control Release. 2007;118:54-58.

22. Couvreur P, Kante B, Roland M, et al. Poly(cyanoacrylate) nanocapsules as potential lysosomotropic carriers: preparation, morphological and sorptive properties. J Pharm Pharmacol. 1979;31:331-332.
International Journal of Nanomedicine

\section{Publish your work in this journal}

The International Journal of Nanomedicine is an international, peerreviewed journal focusing on the application of nanotechnology in diagnostics, therapeutics, and drug delivery systems throughout the biomedical field. This journal is indexed on PubMed Central, MedLine, CAS, SciSearch ${ }^{\circledR}$, Current Contents ${ }^{\circledR} /$ Clinical Medicine,

\section{Dovepress}

Journal Citation Reports/Science Edition, EMBase, Scopus and the Elsevier Bibliographic databases. The manuscript management system is completely online and includes a very quick and fair peer-review system, which is all easy to use. Visit http://www.dovepress.com/ testimonials.php to read real quotes from published authors.

\footnotetext{
Submit your manuscript here: http://www.dovepress.com/international-journal-of-nanomedicine-journal
} 